\title{
Short-Term Earthquake Forecast with the Seismic Sequence Hierarchization Method
}

\author{
Giulio Riga1, Paolo Balocchi² \\ ${ }^{1}$ Geologist, Independent Researcher, Lamezia Terme, Italy \\ ${ }^{2}$ Geologist, Independent Researcher, Modena, Italy \\ Email: giulio.riga@tin.it
}

Received 19 February 2016; accepted 8 May 2016; published 11 May 2016

Copyright (C) 2016 by authors and Scientific Research Publishing Inc.

This work is licensed under the Creative Commons Attribution International License (CC BY).

http://creativecommons.org/licenses/by/4.0/

(c) (i) Open Access

\begin{abstract}
All strong earthquakes are preceded by branching structures having different durations whose development scheme is partly largely predictable because it follows a well organized and recognizable pattern. By using a seismic sequence hierarchization method, this study graphically explains the preparation process of an earthquake, called "branching structure". In addition, criteria apt to distinguish the structures that will produce shocks of average magnitude from strong earthquakes' will be defined. Based on the temporal oscillations of the magnitude values, we explain the procedure for identifying the developmental stages that characterize the energy accumulation stage of the branching structure, in order to early detect the energy release stage's trigger point and obtain information on how it will develop over time. The study identifies also some pre-signals (trigger points) of various magnitudes in the energy release stage, which allows us to early predict the foreshocks and mainshock time position. The method we developed constitutes a truly innovative approach for the earthquake forecasting analysis, which dramatically differs from those developed so far, as it considers the structure of the seismic sequence not only as a magnitude values' oscillation, but also as a sequence of developmental stages that may begin much earlier.
\end{abstract}

\section{Keywords}

Branching Structure, Earthquake, Microsequence, Hierarchization, Foreshock

\section{Introduction}

In some cases the main shock is triggered by one or more lower magnitude early events, called foreshocks, which in turn can trigger additional seismic events. Even a strong earthquake is followed in time and space by groupings of earthquakes with a lower magnitude, called aftershocks, which generally are triggered by the same 
main event.

These aspects related to the aftershocks behavior have been incorporated in the triggering models, where the probability of occurrence of an earthquake in a given time and place is associated with the past history of the earthquakes occurred in the surrounding areas. The most famous model is the short term ETAS (Epidemic Type Aftershock Sequence) [1] [2], where each event, regardless of its magnitude, increases the probability of triggering seismic events in the surrounding areas. In this system, a sequence of aftershocks may be seen as a "trigger cascade” [3] that extends geographical scope of the main initial shock. Conversely, according to our opinion, this approach highlights how the seismic sequence is a seqeunce of energy release and accumulation phases that develop before and after the strongest shocks [4].

A long term challenge consists in determining which earthquakes are connected, directly or indirectly, and in identifying the earthquakes sequences based on causal connection [5]. In the past, various declustering algorithms were proposed to subdivide earthquakes into main shock and aftershocks [6]-[11]. Some of these methods are arbitrary rules characterized by various parameters which jeopardise their objectivity. To solve these issues, a series of methods that seek to evaluate the correlations between the earthquakes has been developed [6]-[11].

We believe that the study and identification of branched structures, without resorting to a physical modeling, can provide valuable information on the evolution of the seismic sequence and on the process of strong earthquake preparation as well as on triggering relationships between earthquakes.

An unquestionably crucial aspect in the analysis of the magnitude values' historical series is the identification of the graph patterns that may help to explain some of the dynamics issues related to the preparation of an earthquake, especially those anticipating an energy release cycle and allow us to identify the critical stages.

For example, the branching structure is a pattern that not only allows us to evaluate graphically the extent of the preparation process of an earthquake but also contributes to outline the past and future evolution of an area's seismicity.

In the initial developmental stages, the branching structures develop slowly, while in the final phases before the energy release stage, the decrease of the magnitude values is very rapid.

These are identified by considering that after an energy shock, the magnitude values gradually decrease up to the trigger point by following a hierarchization process.

Normally, each branching structure is connected with one or more medium-strong earthquakes concerning a certain area. In some cases, after the branching structure's trigger point formation, various orders foreshocks with increasing magnitude may form, which play a crucial role in the preparation phase of an earthquake.

In this case, the energy release stage is characterized by one or more foreshocks as well as by one or more mainshocks [4] [12].

By using the graphic and quantitative analysis, we can learn the developmental stages of the branching structure, assess whether the phenomenon develops according to the expected standard scenario and know early the "hazard degree" (Short-Term Earthquake Probabilities) of the region examined, which heavily depends on space-time relationships between each event [13]. Therefore, the hazard degree is a function of the seismic sequence's developmental stage. If the area is located in the first developmental stages, the hazard degree of the area will be relatively low compared to the last developmental stages where it will feature a greater degree.

The analysis of the seismic sequence structure is performed using: 1) a datasets spatial coverage involving either parts of a region or several countries (from a few tens of $\mathrm{km}$ to thousands of $\mathrm{km}$ ), 2) a magnitude values range of 2.0 - $10 \mathrm{~m}, 3$ ) a depths range of $1-50 \mathrm{~km}$.

\section{Branching Structure Developmental Pattern}

The strongest earthquakes usually trigger aftershocks aimed at re-adjusting the new stress condition resulting from the strongest shock. The aftershocks show a peculiar pattern that develops over time following a hierarchization process identified as branching structure. Such structures are not born equal, but since the first developmental stage we can understand if they will generate a medium or strong earthquake. Indeed, the energy that a branching structure can release in the energy release stage, depends on the number and duration of the developmental stages and the average magnitude of the first developmental stage. The greater the duration, the number of the developmental stages and the average magnitude of the first developmental stage, the greater the released energy.

The entire branching structure preparation process can be divided into three phases: 1) energy accumulation; 2) triggering; 3) energy release (Figure 1) [4]. 
1) Energy accumulation stage. It consists of five developmental stages related to temporal amplitude, magnitude and number of seismic events decreasing over time, which may even last for several years and in some cases constitutes $90 \%$ of the branching structure.

The most energetic shocks take place during the first developmental stage.

2) Trigger stage. It consists of small magnitude shocks organized in an easily detectable micro-sequence called "Double Bottom-Three Small Earthquakes" (DB-3SE). This stage is easily recognizable when the last shock coincides with the point in which the elastic deformation probably reaches its peak local values (trigger point).

3) Energy release stage. During this stage, the energy is released immediately after the trigger point (Flash earthquakes type) or in one or more cycles of increasing amplitude and magnitude (Progressive earthquakes type), characterised by a seismic activity acceleration known as foreshocks [14] [15]. Foreshocks depend on different, still unclear physical mechanisms [16]-[18] and start to develop even hours/years before a strong earthquake

In summary, in the pattern of the branching structure the following apply:

1) The most energetic earthquakes occur, at irregular intervals, during the first part of the energy accumulation stage and in the final part of the energy release phase;

2) The amplitude of the branching structure's developmental stages tends to decrease over time in the energy accumulation stage and to increase in the energy release stage;

3) The energy can be abruptly (Flash earthquakes type) or gradually (Progressive earthquakes type) released, alternating standby with peak activity periods;

4) The energy release stage is activated by small magnitude earthquakes organized in simple DB-3SE microsequences wherein the last shock is the trigger point;

5) In the energy accumulation and release stages, multiple seismogenetic structures, at regional or interregional level, may be involved [19]-[21];

6) The aftershocks and foreshocks contribute to dissipate tectonic energy. Conversely, the cumulative effect of aftershocks increases it while foreshocks' lower it [22].

Figure 1 shows the branching structure's pattern: we see that the first developmental stage (the widest) starts to develop after a strong earthquake, while the subsequent ones are related to the magnitude values decrease over time. The branching structure's trigger point determines the transition from the accumulation to the energy release stage that develops in a shorter time compared to the first.

\section{Methods}

\subsection{Linear Graph}

The graph is one of the most important components in making both long- and short-term predictions process.

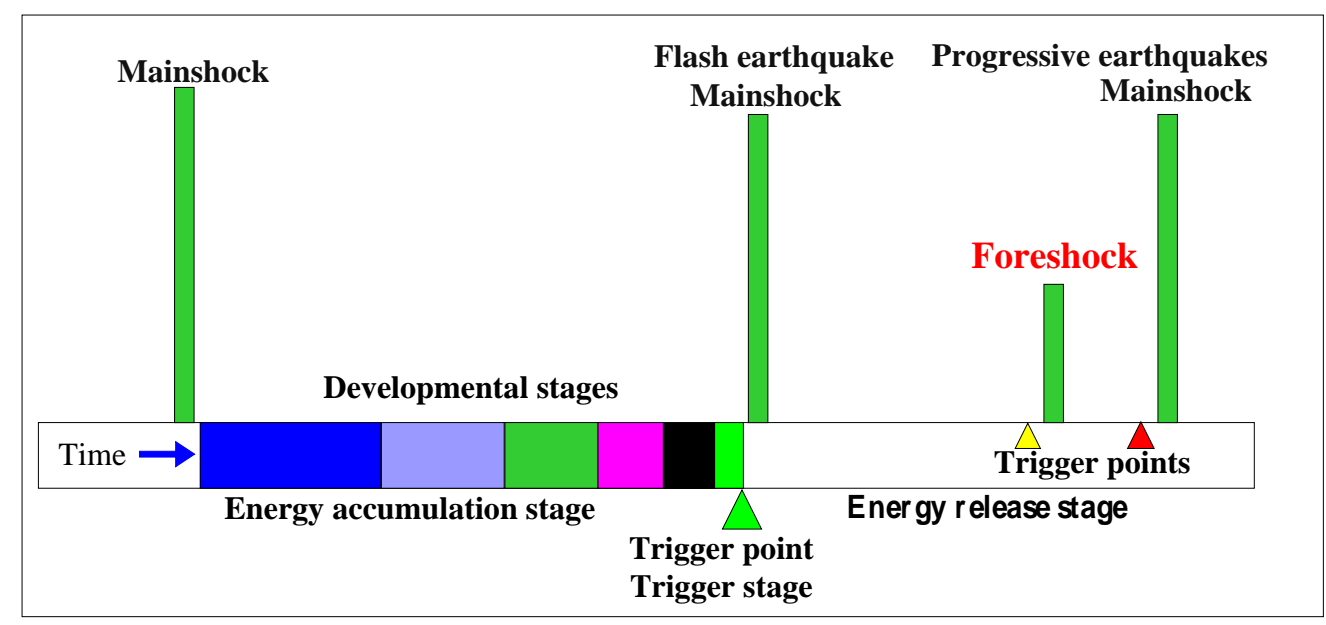

Figure 1. Branching structure's schematic representation. The variously colored rectangles indicate the developmental stages of the energy accumulation stage. 
By representing the magnitude values as a linear graph, we can see how the magnitude values behave in the time window considered, identify the energy accumulation and release stage, detect the premonitory patterns [2], set projections related to future magnitude values and strong earthquakes occurrence times.

The time frame used to process a short term graph can be daily/weekly, while for the medium/long-term is monthly/yearly. Obviously, the wider the reference window, the greater the details shown in the graph.

The line segment that connects two points on the graph shows a local stage, which can be visually interpreted compared to other lines' by means of a coded color.

Figure 2 shows the linear graph of the magnitude values whose reading is quite simple. As we note, the seismic sequence develops by alternating energy release with adjustment stages. Black-colored circles indicate the magnitude points $\mathrm{M}$ in which the reverse of the cyclical adjustment (red-colored lines) and energy (greencolored lines) stages occur. Both the energy release and adjustment stages may consist of one or more seismic events.

Although this type of graph is perfect for a quick and immediate glimpse of the seismic sequence developmental structure, we need to examine it in depth to obtain more detailed information.

For an early identification of the energy accumulation and release stages, we need to join the relative maximum points with a straight line both in case of downward and upward magnitude values (Figure 3).

These straight lines are called dynamic trendlines (magnitude values vary with the time) and clearly indicate the direction in which the values develop, both in the forthcoming and in the long term period.

The upward breaking of the breakout trendline activates the energy release stage, which is characterized by one or more mainshoks (Flash earthquakes type) or by a first foreshock (Progressive earthquakes type) followed a first adjustment shock, which involves the magnitude values' return to somewhere close or below the energy accumulation trendline. Then the values begin to rise again until a second foreshock occurs, which allows us to understand the direction of the energy release trandline.

Typically, although with the Progressive earthquakes type we can record up to four foreshocks, most frequently the pattern consists of two foreshocks and one mainshock.

Besides indicating the ongoing trend, the trendlines temporarily prevent the magnitude values from rising by allowing, in some cases, the early detection of certain levels of magnitude that will be achieved in the future.

Actually, by analyzing the graph shown in Figure 3, we can see how the knowledge of the magnitude levels achieved in points 1 and 2 allows us to calculate the magnitude value in points 3 and 4 of the energy accumulation stage or the magnitude value in point 6 of the energy release stage after the the points 4 and 5 have formed. The point 4 , which is common to the two phases, is the transition point from the energy accumulation and the energy release stage.

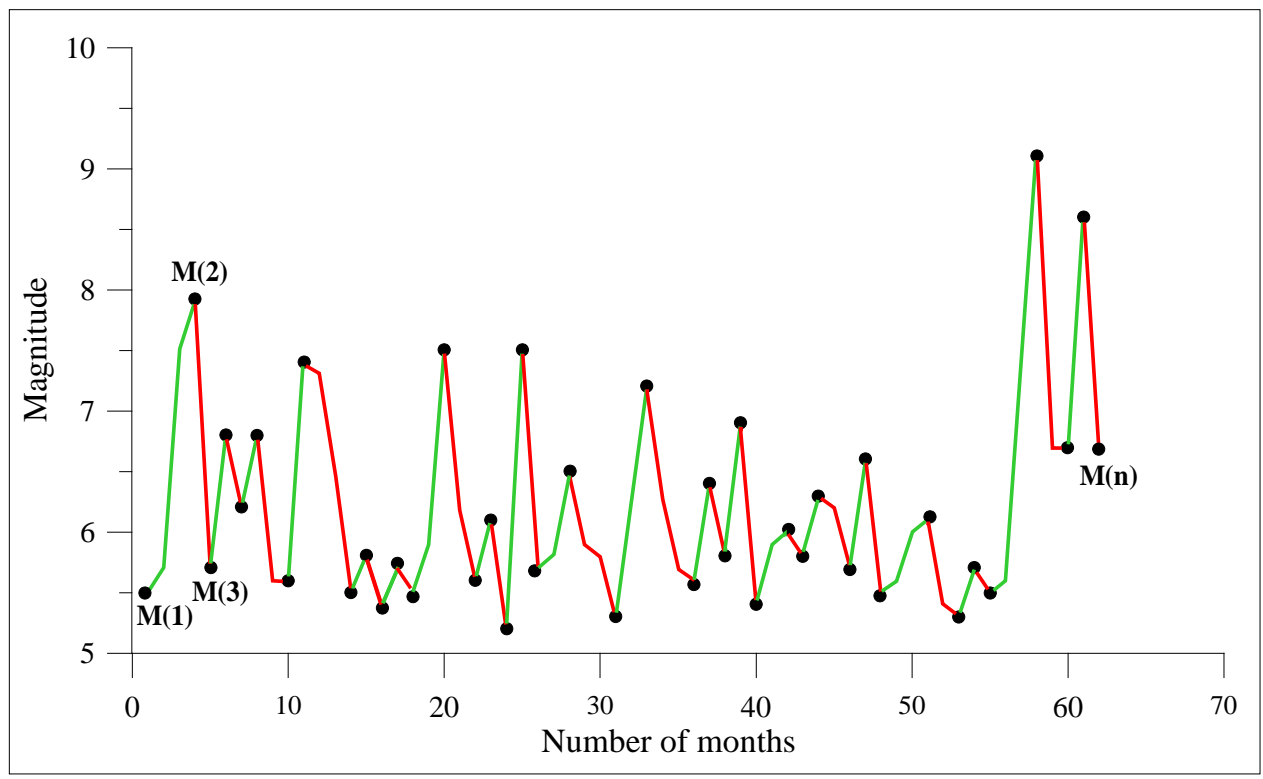

Figure 2. Earthquake linear graph, Japan, 2011. 


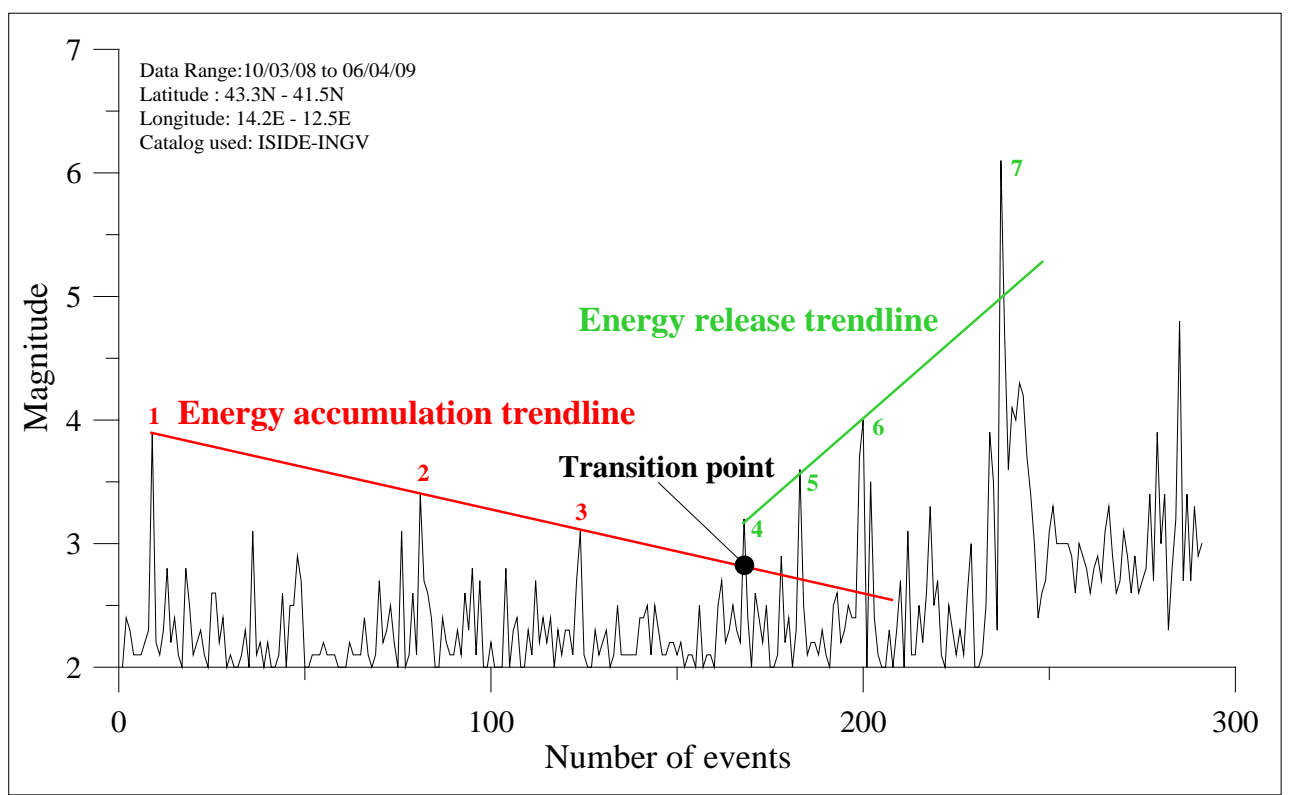

Figure 3. Earthquake linear graph, L’Aquila (Italy), 2009. The red-colored line indicates the energy accumulation trendline, while the green-colored one indicates the energy release trendline. The black-colored circles represent the transition point between the accumulation and the energy release stage.

\subsection{DB-3SE Microsequence-Generated Trigger Point}

Some studies performed on seismic sequences show how earthquakes of any magnitude can trigger other earthquakes [23] [24] that have different triggering properties [25]. Generally, low magnitude earthquakes are greater in number compared to those of higher magnitude: therefore, they collectively can trigger also big seismic events [24] [26] due to the stress in the surrounding area [27]. Through the study of seismic sequence, and with appropriate calculation algorithms it is possible to distinguish the seismic events triggered by the background activity [8].

By analyzing the magnitude values trend occurring before the start of an energy release stage, we can identify a small shocks microsequence that may be used for the prediction of immediate and short term medium/high magnitude earthquakes. In fact, the energy release stage's trigger point coincides with the third shock in the DB-3SE microsequence, which consists of three, small magnitude shocks and is a very reliable stage-reverse signal.

Once completed the last phase of the branching structure's energy accumulation stage, in order to locate the first trigger point and the following ones, in the case where the energy release stage develops according to a Progressive earthquakes-type pattern, it is necessary that the magnitude values of the shocks that make up the temporary DB-3SE microsequence (Figure 4(a)) meet the following conditions:

$$
\begin{aligned}
& M(n-1) \leq M(n)>M(n+1) \\
& M(n-1)<M(n) \geq M(n+1)
\end{aligned}
$$

and

$$
M(n)<M(n-2)
$$

where $M(n)$ is the second shock's magnitude value, while $M(n-1)$ and $M(n+1)$ are the magnitude values of the first and third shock, respectively.

For the completion of the DB-3SE microsequence, we need a subsequent shock whose magnitude must be:

$$
M(n+2) \geq M(n) \text { (Figure 4(b)). }
$$

Figure 4 shows both temporary and final DB-3SE microsequences, while the red-colored triangle indicates the the energy release stage's trigger point. 
The trigger point coincides with the $M(n+1)$ magnitude shock in the DB-3SE microsequence, while the subsequent final shock often corresponds to a more energetic event (foreshock or mainshock). Figure 5 shows the four possible patterns of the final DB-3SE microsequence, depending on the magnitude values of the three shocks which it consists of (red-colored circles).

\subsection{Seismic Sequence Hierarchization}

A seismic sequence may be considered as a succession of cyclical shocks with variable amplitude over time where events occur following a particular hierarchization process during which each earthquake can generate several earthquakes [28].

The herarchization process's basic concept is that a source event (strong earthquake), can generate seismic events of decreasing magnitude during the energy accumulation stage. In essence, these seismic events related to the source event and classified as secondary [29], may also, in turn, generate other minor seismic events, thus triggering a process that continues up to the energy release phase's trigger point and may end after a strong event.

The number of seismic events included in a seismic cycle (adjustment and energy release stages) depends on the seismicity bottom value (i.e., the minimum value in the analyzed magnitude values' time series). The greater the bottom value, the smaller the number of events the cycle will consist of.

The use of a herarchization process in the seismic sequence analysis, allowed us to develop a graph pattern that effectively contributes to outline the past and future development of the seismic sequence. In fact, all the adjustment and energy release stages included in a seismic sequence constitute the "seismic grid", whose development is probably related to various mechanisms involving, among the others, the lithospheric rocks' deformation process and the interaction between the different seismogenetic structures on regional or interregional scale.

The seismic grid can be represented by some geometric entities, such as the "seismic nodes", i.e. the contact points between the two phases, the "reverse phase nodes" that are the transition points between the adjustment and the energy release stage, and, finally, the energy release (r) and accumulation (a) "seismic branches" that make up a seismic cycle (Figure 6).

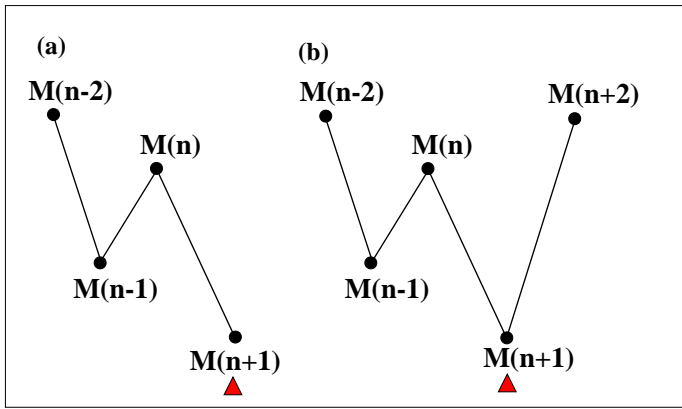

Figure 4. Schematic representation of DB-3SE microsequence: (a) temporary microsequence, (b) final microsequence.

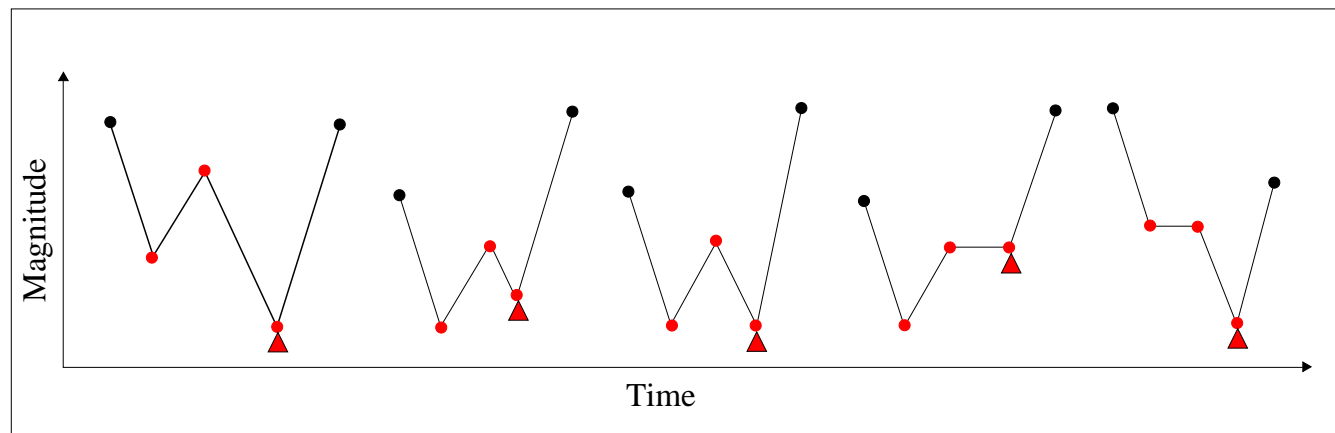

Figure 5. Schematic representation of microsequence DB-3SE. The red-colored circles indicate the "DB-3SE" microsequence while the red-colored triangle indicates the trigger point. The black-colored circles represent the microsequence's initial and final shocks. 


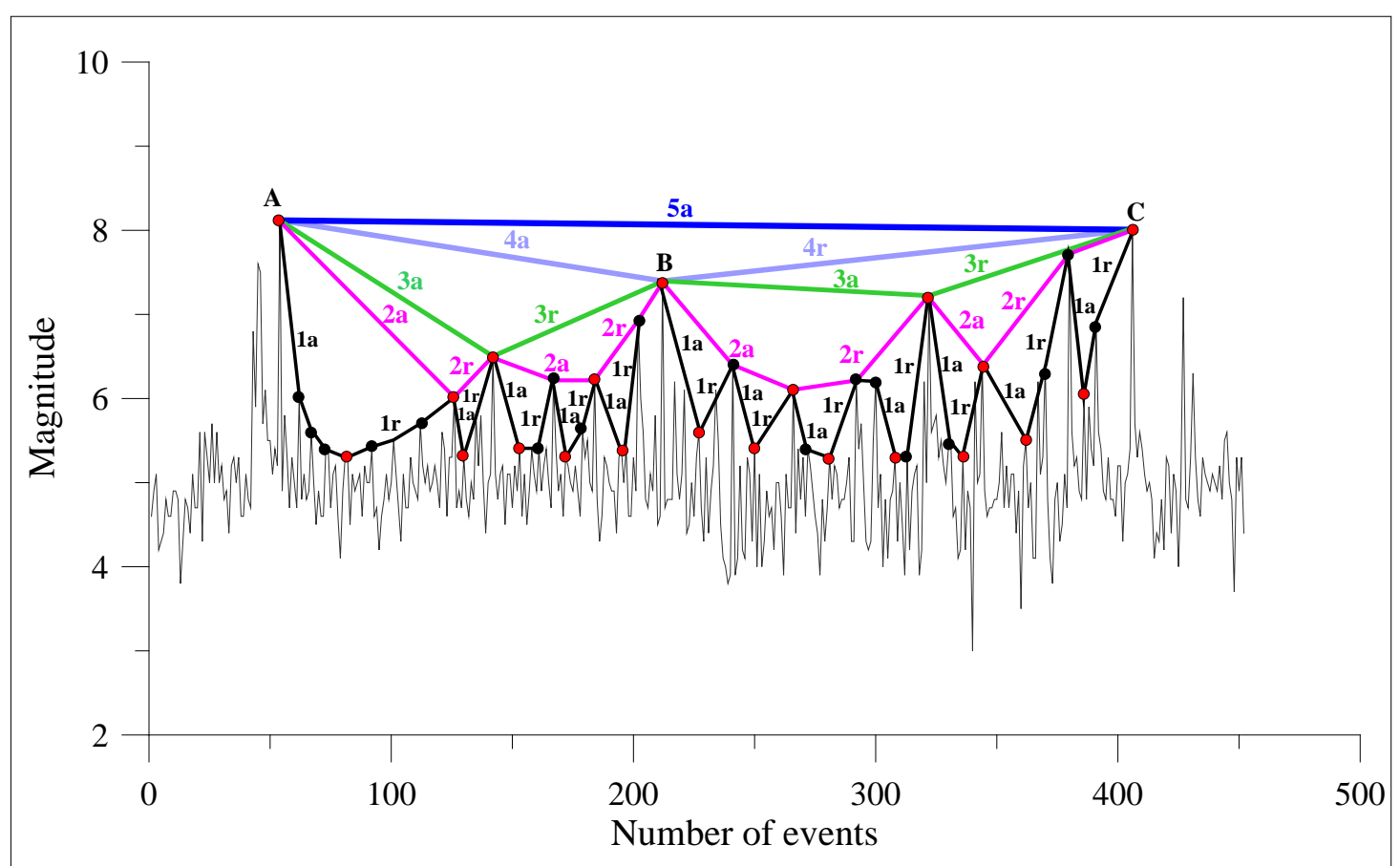

Figure 6. Schematic representation of the seismic sequence hierarchy. Each circle represents a seismic node, while the red-colored ones are the reverse phase nodes. The lines of different thickness and color are the earthquakes' branches. The higher order AC branch represents the higher order seismic cycle consisting of the energy accumulation (4a) and release (4r) stages. We see that the magnitude value is greater in the reverse nodes A, B and C related to higher order seismic branches.

As to the branches arrangement, the seismic grid can be arranged by applying a hierarchization method that consists in assigning an order number to the various branches that make up the seismic grid according to the following rules:

- One or more branches of $\boldsymbol{n}$ order energy accumulation followed by one or more $\boldsymbol{n}$ order energy release branches result in a $\boldsymbol{n}+\mathbf{1}$ order branch (the hierarchical order increase occurs);

- The magnitude value of the "reverse phase nodes" increases as the cycle order increases;

- The higher order branch (5A) is the higher order seismic cycle.

In general, in the various cycles we can observe the proportionality principle, i.e. the higher is the order of the energy accumulation seismic branch, the greater the expected magnitude in the energy release stage.

Moreover, the smaller the inclination of the higher order energy accumulation branch, the greater the time or the number of shocks needed for the energy accumulation stage to be completed.

The end of a $(n)$ order seismic cycle usually takes place after the amplitude of the $(n-1)$ order energy release branch has reached a value equal to the amplitude of the $(n-1)$ order energy accumulation branch plus $50 \%$ of it.

We can obtain more accurate data on the developmental level of a seismic grid by removing the seismic branches related to the energy release stage (Figure 7). The main branching structure obtained (S,A,B,C,D) retains the remote seismic events and the pre-earthquake seismic context's effects which we can obtain information from in order to determine its future development.

Note how the various orders seismic branches begin to form after a strong earthquake (source node S-source node). and are related to the magnitude values decrease over time. We also see how from the early stages of the seismic grid formation, it is possible to identify the branching structures that, depending on higher order seismic branch, will produce shocks of average magnitude or strong earthquakes in the energy release stage (the greater the order of the first seismic branch that originates from a source point, the greater the expected magnitude). Actually, the branching structures are not all the same but may take different appearances: their duration and the energy they release in the energy release stage, depend on the magnitude values of higher order branch and the time arrangement of low order subsequent seismic branches. 


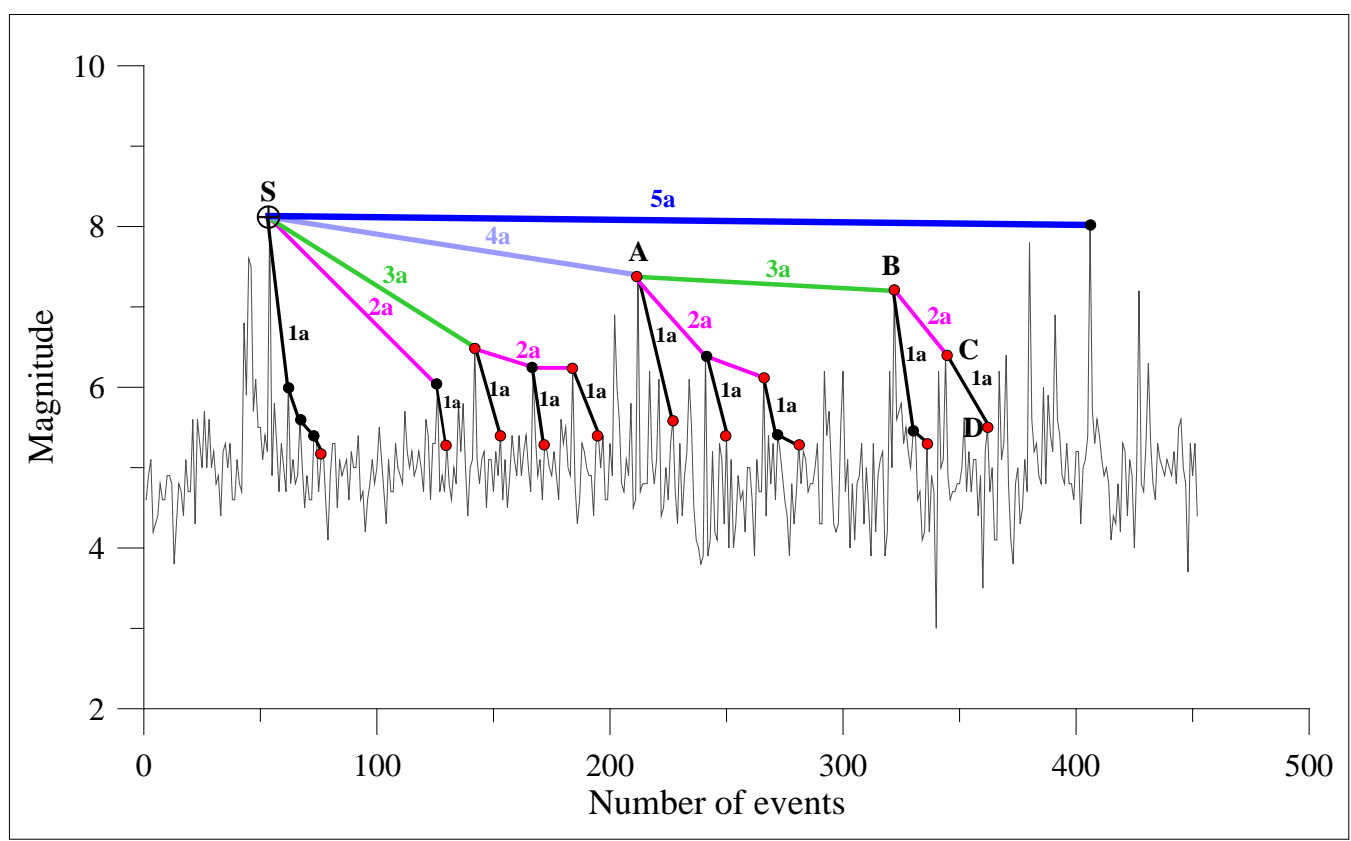

Figure 7. Schematic representation of the branching structure obtained by removing the energy release stage's seismic branches. The red-colored circles represent the reverse node of all the branching structure identified, while the letters S, A, B, $\mathrm{C}$ and $\mathrm{D}$ indicate those in the main branching structure (the point $\mathrm{S}$ is the source-point).

A branching structure is perfectly hierarchical when each ( $n$ ) order branch is followed by another that features an immediately lower order $(n-1)$. If this is not the case, a seismic anomaly occurs. In addition, when the seismic branches' order decreases, the time or the number of shocks that we need to wait for the trigger stage to be formed, which precedes the energy release stage, decreases as well.

This means that only after the formation of the (1a) first order branch, the branching structure enters a critical stage where the DB-3SE microsequences generate the trigger point that can be profitably used for short term forecast.

\subsection{Graphical Procedure for Branching Structures Identification}

The long-term, monthly hierarchization of the seismic sequence (from 1973 to 2004) in Indonesia, which consists of any single maximum magnitude value monthly recorded by assuming a bottom value of $2.0 \mathrm{M}$, can be implemented by identifying the reverse nodes and following the multi-step procedure as follows:

1) Locate the first order reverse nodes $P 1(n)$ that meet the following condition:

$$
M(n-1)<M 1(n) \geq M(n+1)
$$

where, $M 1(n)$ is the value of the first order reverse node magnitude and $M(n-1)$ and $M(n+1)$ are the magnitude values of seismic events that precede and follow the first reverse seismic node (Figure 8), respectively;

2) Locate the second order reverse nodes $P 2(n)$ that meet the following condition:

$$
M 1(n-1)<M 2(n) \geq M 1(n+1)
$$

where, $M 2(n)$ is the magnitude value of the second order reverse node and $M 1(n-1)$ and $M 1(n+1)$ are the magnitude values of the first order reverse nodes that precede and follow the second order reverse node;

3) Draw the black-colored line joining the second order seismic nodes;

4) Repeat the same procedure to locate the second, third and fourth order reverse nodes (magenta-, green- and pastel blue-colored lines) (Figure 9);

5) Locate the line joining the second order reverse nodes (black-colored lines), P2m(n) second order minimum reverse nodes whose magnitude must comply with the following condition:

$$
M 2(n-1) \geq M 2 m(n)<M 2(n+1)
$$




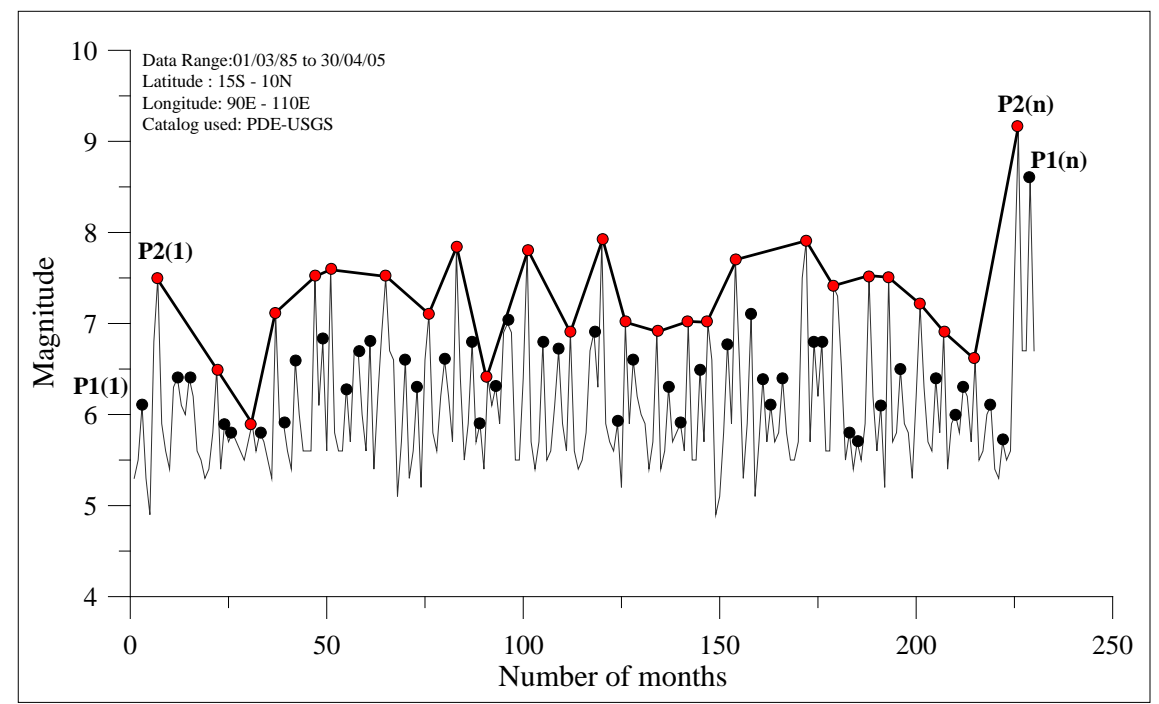

Figure 8. Earthquake's seismic sequence, Indonesia, 2004. Representation of the first order reverse nodes (black- and redcolored circles) and the second order reverse nodes (red-colored circles).

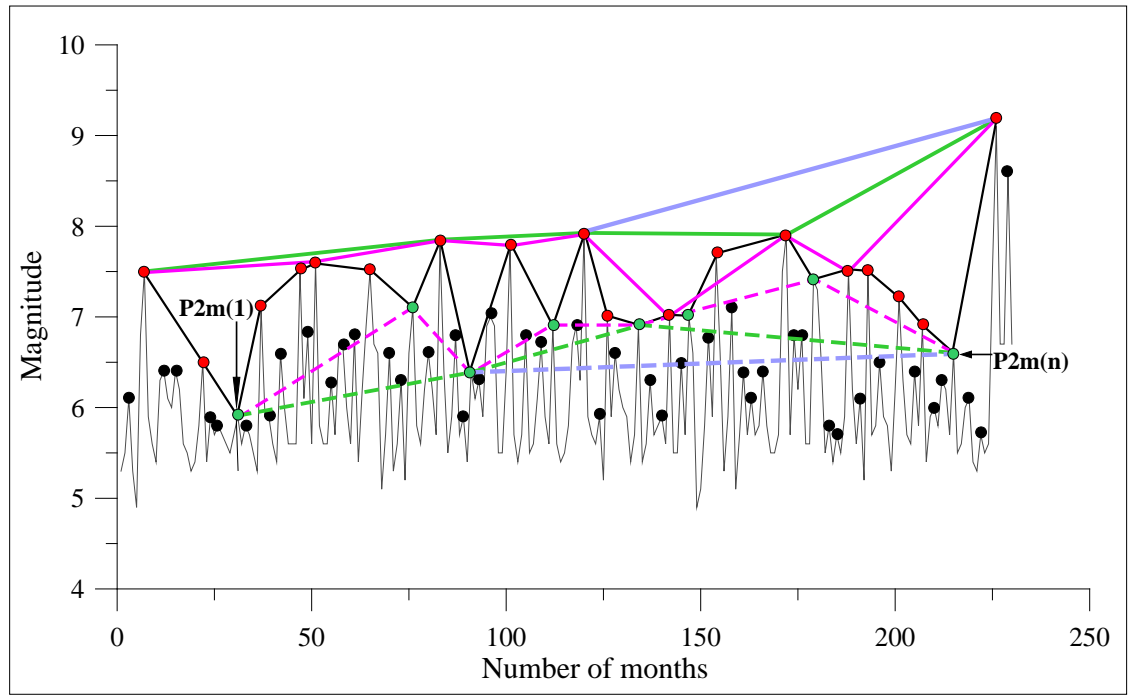

Figure 9. Fluctuation of various orders reverse nodes. The solid-colored line indicates various orders seismic branches that join the maximum reverse nodes, while the dashed colored lines indicate various orders seismic branches that join minimum reverse nodes.

where $M 2 m(n)$ is the magnitude value of the second order, minimum reverse node and $M 2(n-1)$ and $M 2(n+1)$ are respectively the magnitude values of the second order reverse nodes that precede and follow the second order minimum reverse node (Figure 8);

6) Join the second order minimum reverse nodes with a line (magenta-colored dashed line);

7) Repeat the same procedure to draw higher order green, pastel blue and blue-colored dashed lines.

In order to identify various order branching structures we need to remove from the graph the energy release seismic branches as well as the descending seismic branches that join the minima (dashed lines).

Moreover, we need to identify the triggering stages (TS) of the branching structures, by drawing the line joining the lower extremity of the first order seismic branch with the trigger point generated by the DB-3SE microsequence (light green — colored line).

The colors assigned to the seismic branches and triggering stage let us determine the development status achieved by the various order branching structures. 


\subsection{Results}

Figure 10 displays several various orders branching structures (BR1, BR2 and BR3) that have developed over time. The greatest (BR3) consists of an energy accumulation stage characterized by four developmental stages and a flash earthquake-type energy release stage featuring a mainshock with a magnitude of 9.1 Mw on 26-12-2004.

During the branching structures' early development and completion stages, several various orders trigger points were generated. In the graph, the yellow-colored triangles, placed at the extremities of the minimum ascending branches (magenta-colored dashed lines), represent the first order trigger point, while the red-colored triangles indicate the second order ones generated by the DB-3SE mirosequence.

In the lower branching structure (A,B and C), we note the abnormal formation of a fourth order branch that generated a trigger point in the node $\mathrm{C}$ (yellow triangle) just before the mainshock.

The same procedure can be used to locate the greater magnitude aftershocks in the energy accumulation phase.

Figure 11 shows the branching structures that have developed after the earthquake occurred Indonesia on 26 December 2004 (source node).

In particular, we observe a fourth order branching structure, which on 28 March 2005 the first significant aftershock developed from with a magnitude of $8.6 \mathrm{Mw}$, that originated the first, fourth order seismic branch.

\subsection{Modified Aroon Oscillator}

The Aroon oscillator [30] was modified so as to be able to obtain adequate information on the type of branching structure (primary or secondary) being formed and on the developmental stages' hierarchization degree (whether a branching structure is located in an accumulation or release stage).

The oscillator features a red line that indicates the energy accumulation stage and a green line indicating the energy release stage (Figure 12). The scale used by the oscillator ranges from $0 \%$ to $100 \%$.

When the values are increasing, the oscillator indicates an energy release stage in progress, while when the values are decreasing, it indicates that an accumulation energy stage is being formed. The farther the oscillator from the value of $50 \%$, the stronger the current phase. Usually, the oscillator is decreasing if seismic branching with the same order is subsequent to each other.

There are other basic situations for interpreting the indicator:

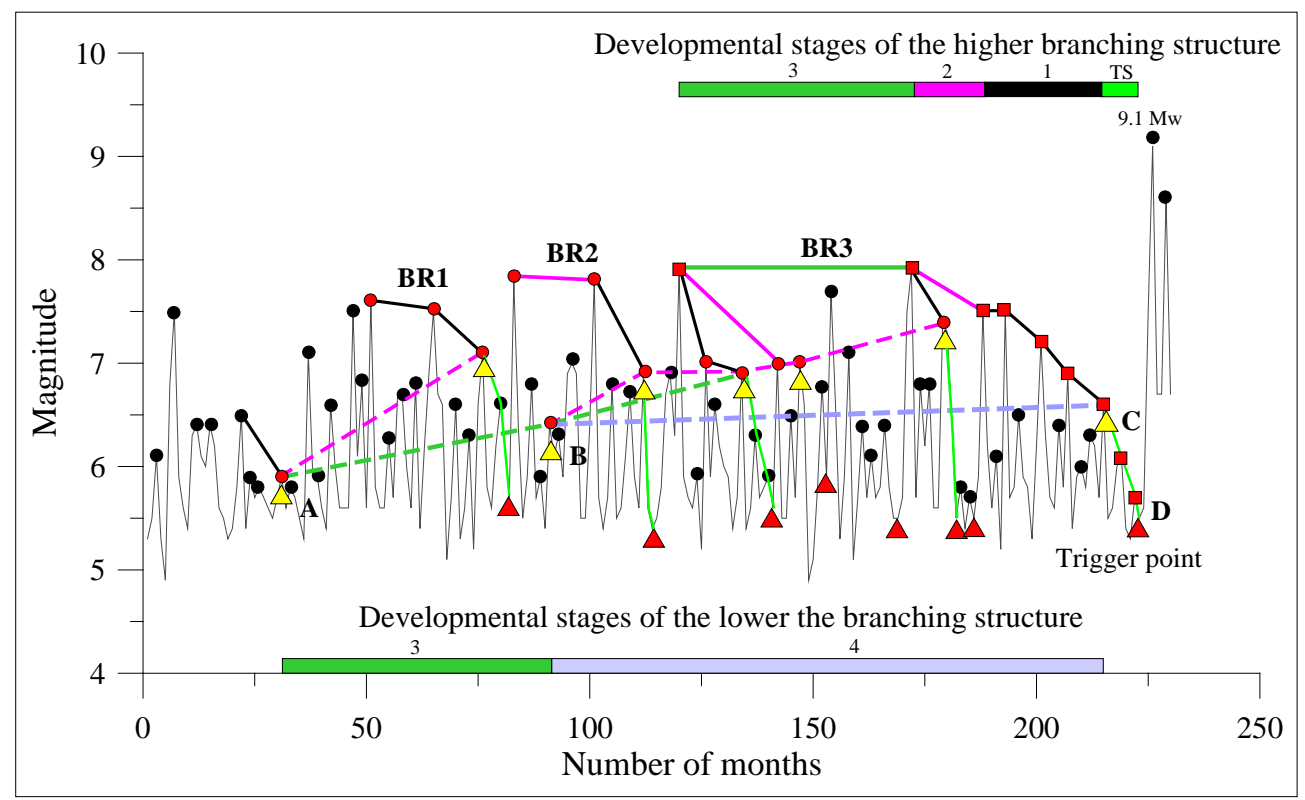

Figure 10. Earthquake's seismic sequence, Indonesia, 2004. The red colored squares show the higher order nodes of the branching structure, while the red-colored circles indicate the lower order ones, and the yellow- and red-colored triangles indicate the first and the second order trigger points, respectively. The upper part of the graph shows the developmental stages of the higher branching structure, while in the lower part the developmental stages of the lower branching structure less are reported. 


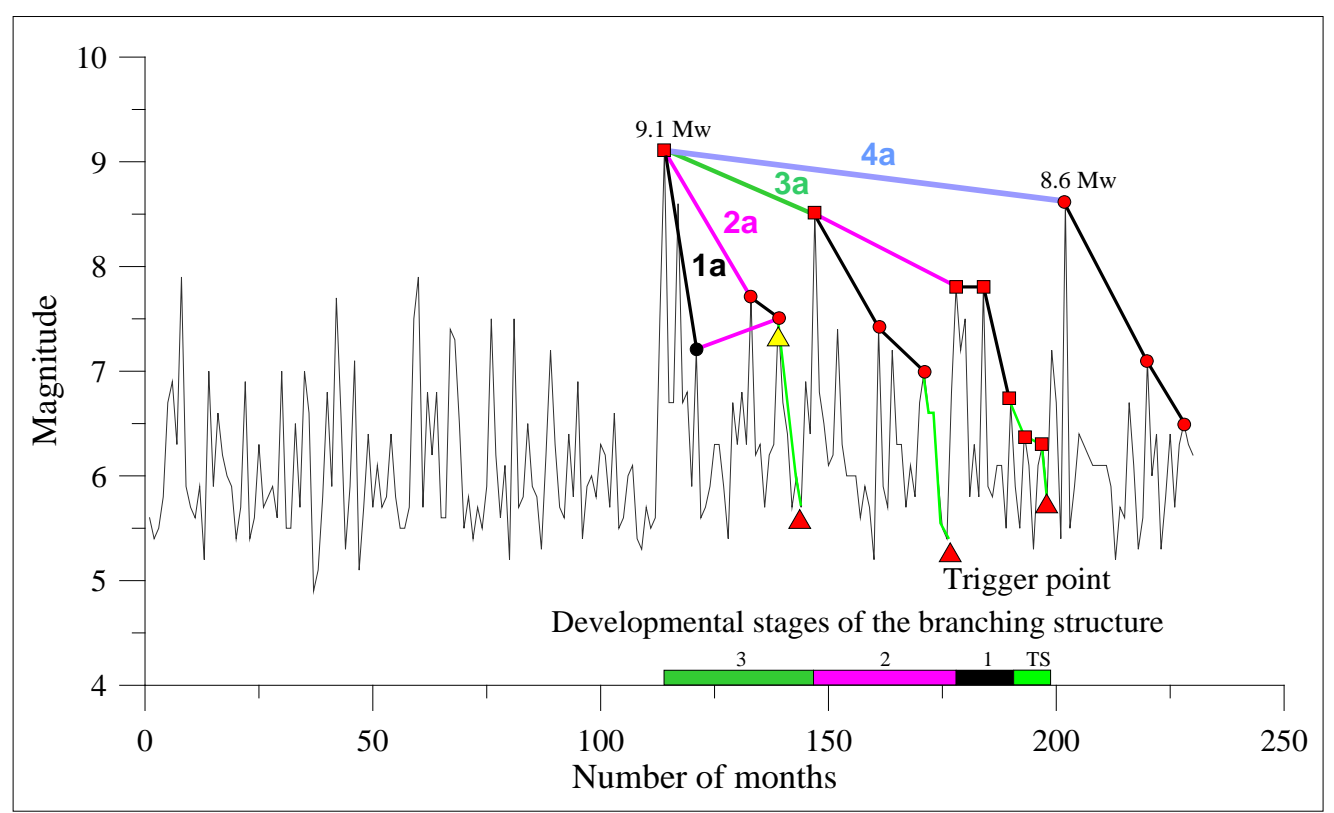

Figure 11. Earthquake's seismic sequence, Indonesia, 2004. Pattern of the branching structure related to the energy accumulation stage. The red-colored square indicates the nodes of the higher order branching structure, while the yellow and red triangles indicate the first and second order trigger points, respectively. The lower part of the graph shows the developmental stages of the branching structure associated with the first significant aftershock.

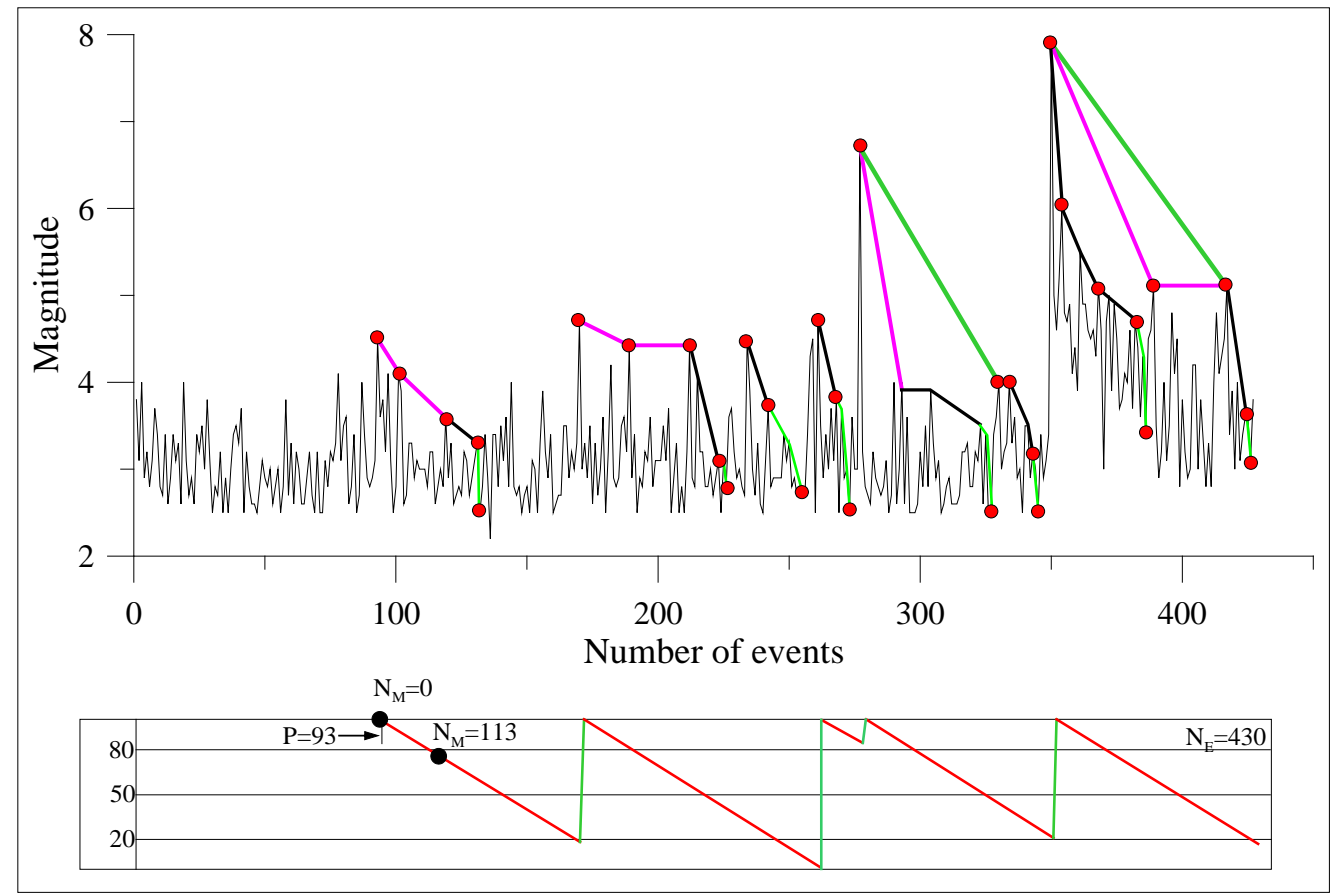

Figure 12. Schematic representation of modified Aroon oscillator. The red-colored lines indicate the energy accumulation stages, while the green lines indicate the energy release stages. The black-colored circles indicate the calculation points.

1) When the oscillator decreases from $100 \%$ to $80 \%$, it indicates the beginning of an energy accumulation stage.

2) When the oscillator decreases $20 \%$ to $0 \%$, it indicates that energy accumulation stage is being completed.

3) When the oscillator increases from $80 \%$ to $100 \%$, it indicates that the energy release stage is being strengthened;

The formula to calculate the modified Aroon oscillator is the following: 


$$
A R=\left[\left(P-\left(N-N_{M}\right)\right) / P\right] \cdot 100
$$

where $P$ (reference period) is the number of seismic events, days or months from the beginning of the seismic sequence to the first reference absolute or relative maximum value (mainshock or average magnitude shock), $N$ is the oscillator's calculation point in relation to the beginning of the seismic sequence and $N_{M}$ is the number of seismic events, days or months from the absolute or relative maximum value that has formed during the reference period with respect to the beginning of the sequence.

Usually the period $P$ must be such that the already formed source points coincide with the points at which the oscillator reaches $100 \%$ (default $p=42$ ).

The algorithm provides for a calculation cycle from $N=P$ up to $N_{E}$ (total number of events in the seismic sequence) in which we need to establish the position of the reference period maximum with respect to the origin of the seismic sequence through a second internal cycle of calculation from $N_{M}=N-(P-1)$ up to $N$, and subsequently calculate the modified Aroon oscillator.

For example, if the period consists of 93 events and the maximum $\left(N_{M}\right)$ and the calculation point $(N)$ of the oscillator are equal to zero, we will have $A R=[(93-(0-0)) / 93] \cdot 100=100 \%$. If the maximum $\left(N_{M}\right)$ occurred in 93 past events and the oscillator's calculation point is $N=113$, then the oscillator's value is

$A R=[(93-(113-93)) / 93] \cdot 100=78 \%$.

\subsection{Maximum Magnitude Assessment Procedure}

Figure 13 shows the branching structures' patterns, which have been identified in the Japan's monthly seismic sequence from 1973 to 31 August 2015.

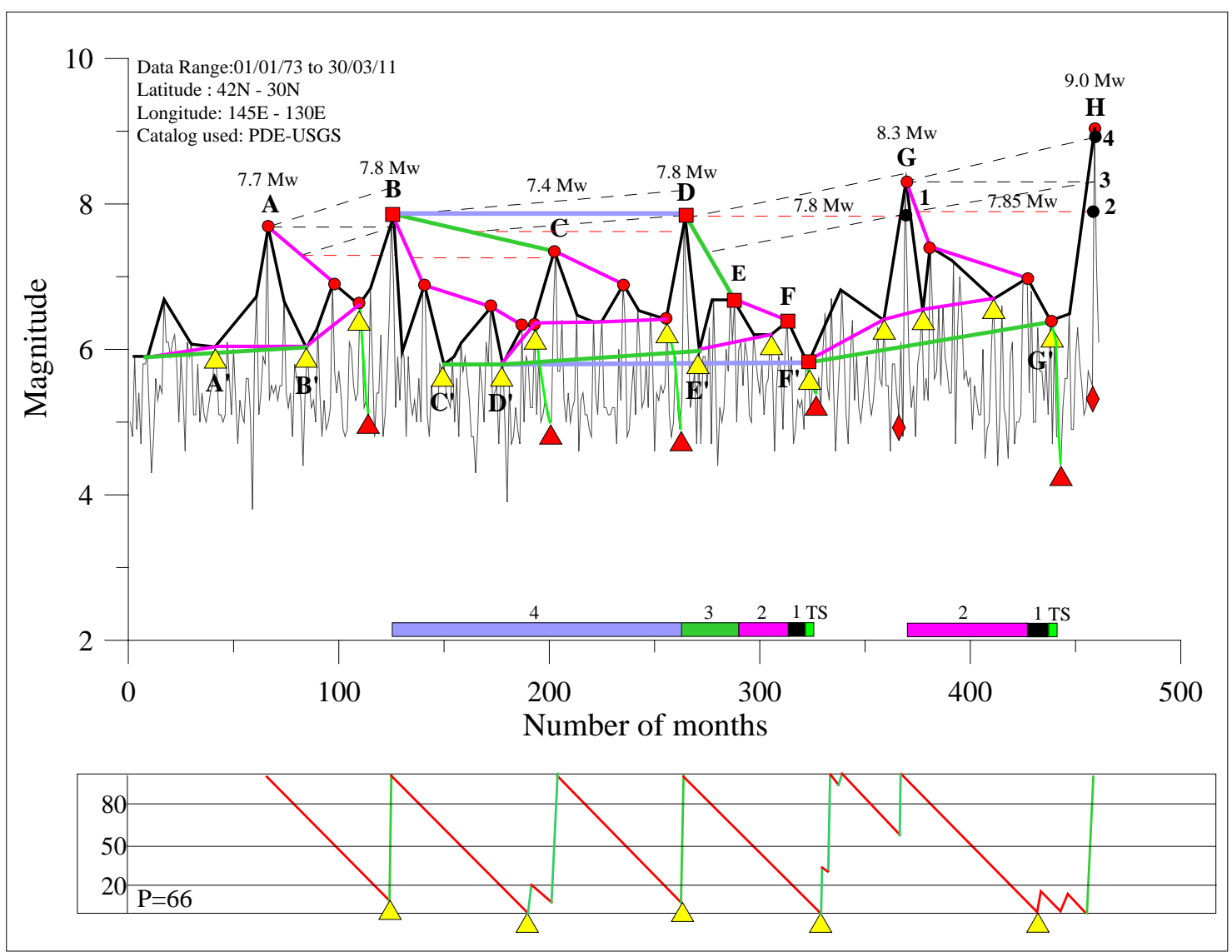

Figure 13. Japan's monthly sequence. The red-colored squares indicate the higher order nodes of the branching structure of higher order, the red circles the minor order ones, while the red and triangles and diamonds indicate the first, second and third order trigger points, respectively. In the lower part of the graph the modified Aroon oscillator is displayed. 
The red squares indicate the main branching structure's nodes, while the red circles indicate the minor branching structures' ones.

The letters A, B, D, G and $\mathrm{H}$ denote the higher seismic nodes that correspond to the strongest earthquakes recorded in the time interval considered, while the letters A', B', C', D', E', F' and G' are the nodes relating to minor, second order ascending seismic branches included in the lower branching structure that correspond to first order trigger points (yellow-colored triangles).

The red-colored triangles and diamonds, generated by the DB-3SE microsequence upon the higher branching structure's completion and during the energy release stage, indicate the second and third order trigger points.

The development dynamics of the branching structure suggests to compute the magnitude value of the strongest earthquake in correspondence with the highest order branch.

From the mid-point of the higher order branch (point 1) included in the branching structure that preceded the earthquake occurred on 11 March 2011, we need to draw an horizontal, dashed line extending to the right up to the calculation point of the expected magnitude value (point 2). The intersection of this half line with the vertical line passing through the set calculation point, provides the minimum magnitude value (red-colored dashed line).

To calculate the maximum value we need to draw a horizontal dashed line from the point $G$ that extends to the right up to the calculation point (point 3 ). Then we join the point 3 with the mid-point of the 2 order branch (point 1); from the point $\mathrm{G}$ we finally draw a parallel line to the segment 1 - 3 (black-colored dashed line).

The intersection of this parallel line with the vertical line passing through the calculation point, gives the maximum magnitude value (point 4).

We should however bear in mind that not always these minimum and maximum values are accurately reflected in the energy release stage. In the case where the range between the minimum and the maximum value is very wide, we can consider the mean value between the minimum and maximum values computed as the maximum value.

Based on Figure 14, which shows in details the energy release stage of the big earthquake occurred on 11 March 2011, with a magnitude of $9.0 \mathrm{Mw}$, we can formulate some interesting considerations about the characteristics of the higher and lower branching structures that preceded the earthquake.

First, we can observe that the first order trigger point coincided with the extremity of the third order lower seismic branch (green line) and that the second and third order trigger points (red box-included red triangle and diamond) that preceded the mainshock were generated by the DB-3SE microsequence.

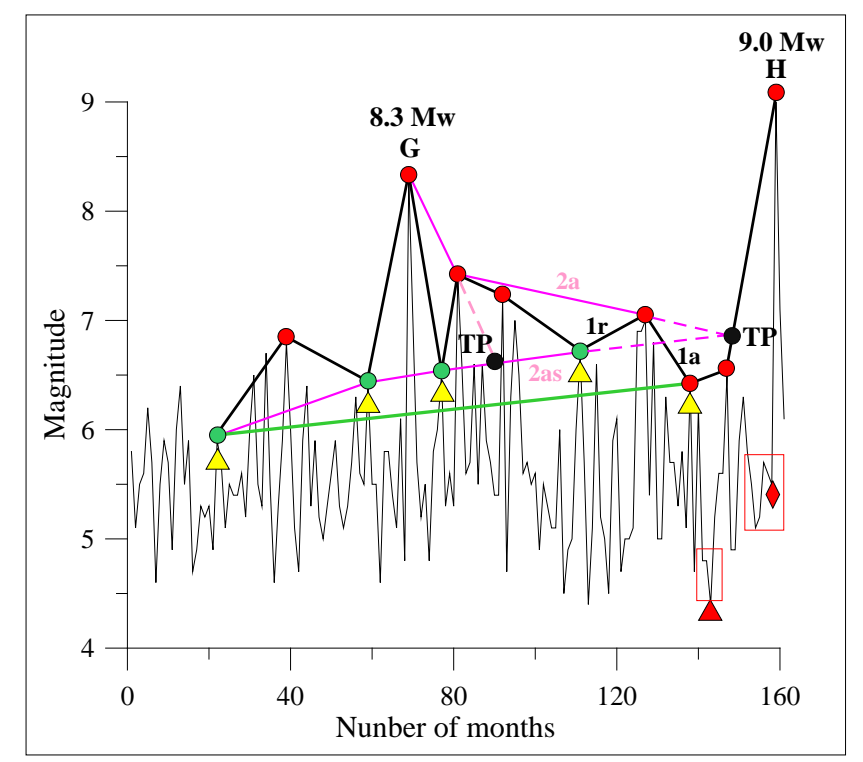

Figure 14. Energy release stage (Japan's monthly sequence). The red circles indicate the higher order nodes of the branching structure, the green circles represent the lower order ones, while the yellow and red triangles and the red diamonds indicate the first, second and third order trigger points. The black-colored circles indicate the trigger points generated by the intersection between the higher second order branches of energy accumulation (2a) lower and ascending magenta-colored ones (2as). 
In addition, by means of a simple graphic procedure we identified a further trigger point (TP) generated by second order higher branches of the energy accumulation stage (2a) and lower ascending, magenta-colored branches (2as). In this case, the next DB-3SE microsequence (red diamond) represents a second order trigger point.

If the TP falls before the lower extremity of the first order energy accumulation branch (1a), we consider the latter as the trigger point.

Figure 15 shows the branching structures that have developed in the time interval between 1973 and 31-08-2015 in the Southern California's seismic sequence. In particular, we note the well hierarchized branching structure that has generated the earthquake occurred in Landers on 28-06-1992, with a magnitude of $7.3 \mathrm{Mw}$, which consisted of two developmental stages with the origin in the node B (source).

The TP was followed by first, second and third order trigger points. The maximum magnitude value calculated with the graphical method starting from the second order branch's mid-point was close to the one recorded.

The Landers earthquake has subsequently generated a third order branching structure (C,E,F,G,E') from which, after the second order trigger point, a hierarchical abnomaly has developed in the node $\mathrm{H}$ (seismic branches FH-HF') consistinting of a third order branch directly followed by a first order one. The energy release stage that followed was of Progressive earthquakes-type, where the strongest aftershocks, including the $7.2 \mathrm{Mw}$ ones occurred on 04-04-2010 (Baja California, Mexico), were preceded by first and second order trigger points (yellow- and red-colored triangles). The TP was generated shortly before the strongest shock and after the second order trigger point. Subsequently, the earthquake on 04-04-2010 has activated a new branching structure characterized by a first, fourth order final seismic related to the energy accumulation ( CD branch).

The minimum temporary magnitude value associated with this seismic bomb developmental phase is $7.15 \mathrm{M}$.

\section{Examples of Branching Structures}

The examples shown in Figure 16, processed from the data in Table 1, show the main branching structures

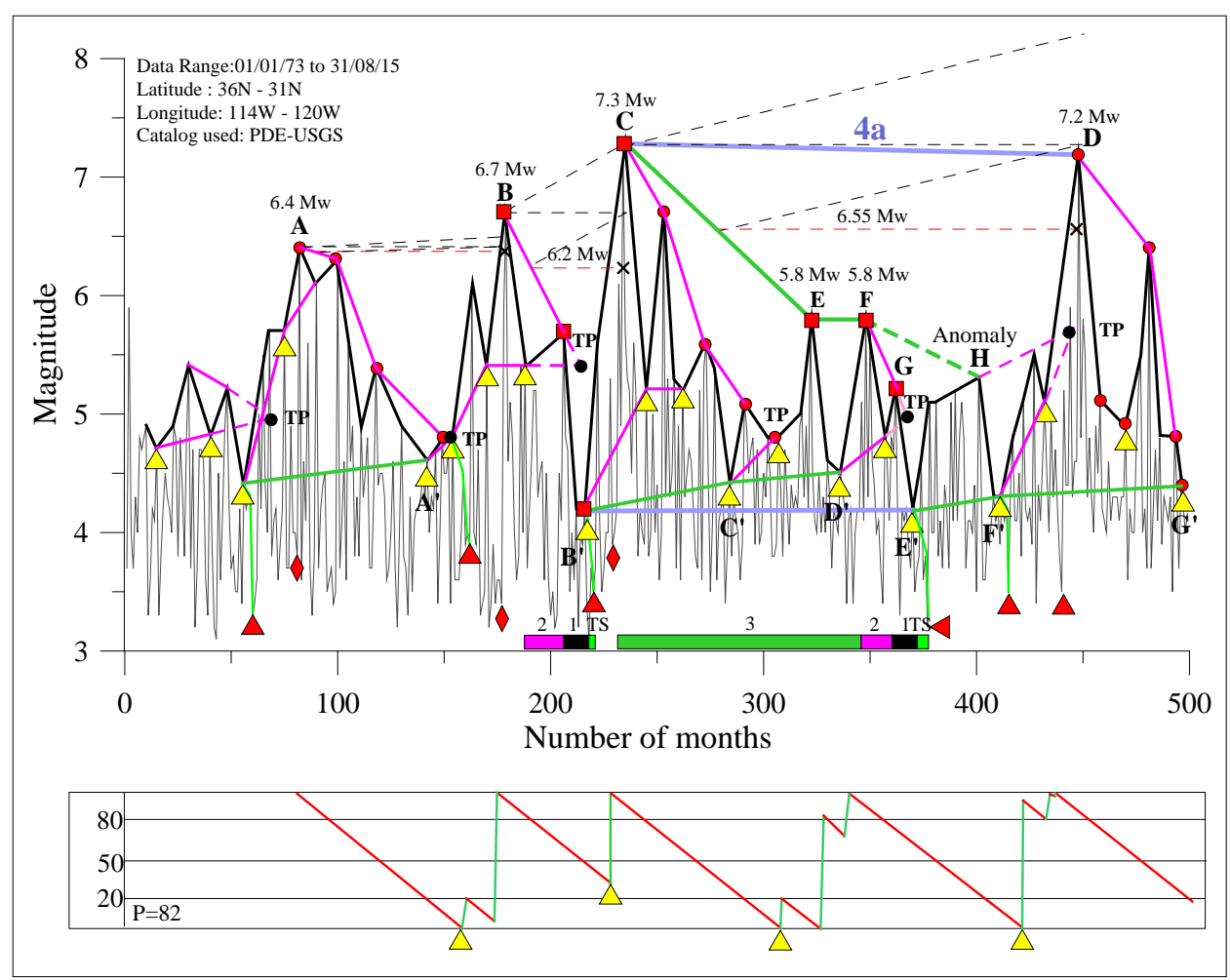

Figure 15. Southern California (US)'s monthly sequence. Red colored squares represent the higher order branching structure nodes, red circles the minor order ones, while the yellow and red triangles and the red diamonds indicate the first, second and third order trigger points, respectively. In the lower part of the graph developmental stages of the main branching structure are reported. 

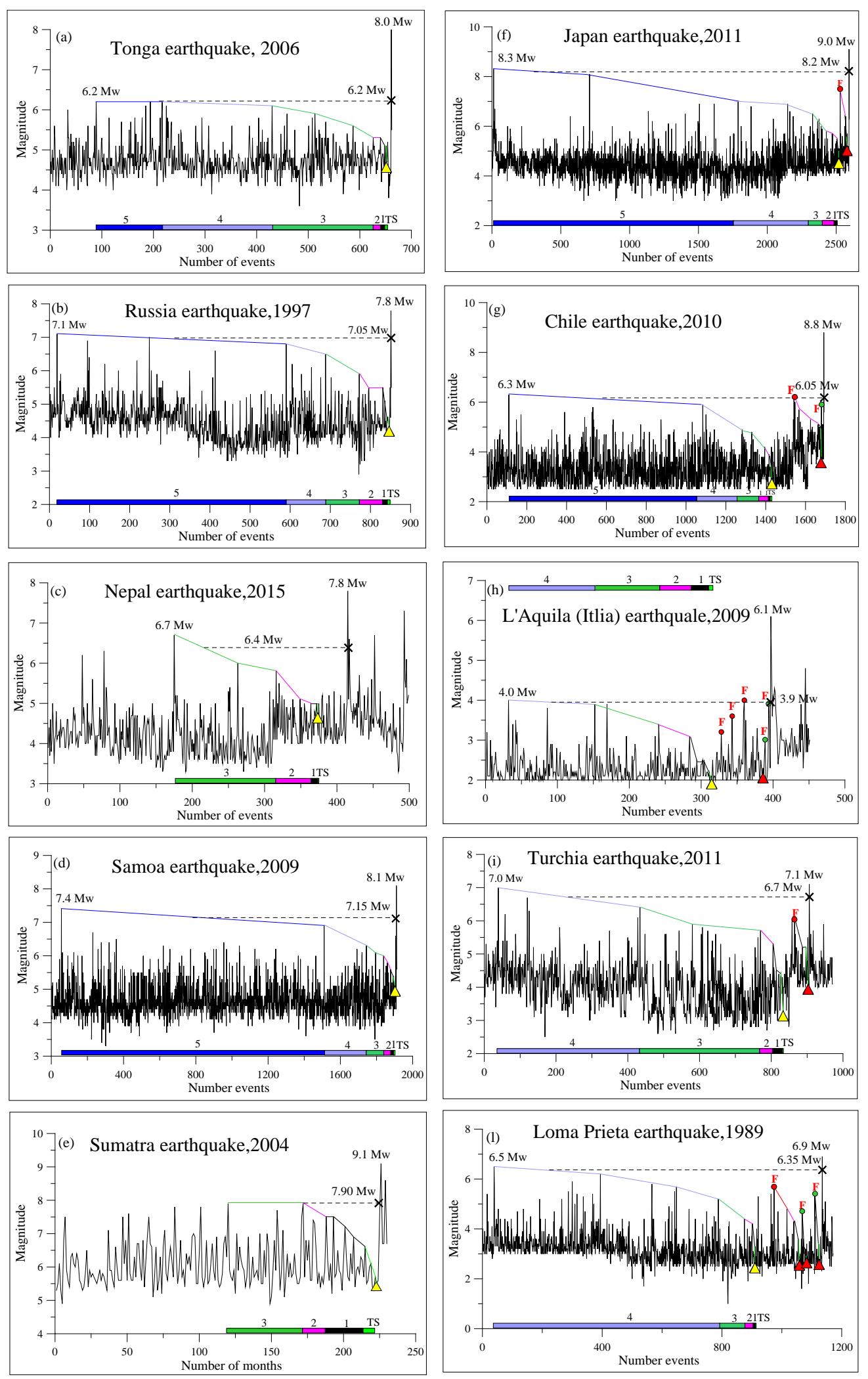

Figure 16. Examples of branching structure. The colored lines indicate the developmental stages, the letter " $F$ " the foreshocks, the red circles the first order foreshocks and the green circles the second order foreshocks. The yellow and red triangles indicate the rigger points of main and secondary branching structures. 
Table 1. Earthquakes, date range, coordinates of the region analyzed and catalogue used.

\begin{tabular}{ccccc}
\hline Earthquakes & Date Range & Latitude & Longitude & Catalog Used \\
\hline L'Aquila-Italy & $27 / 04 / 2007-06 / 04 / 2009$ & $43.2 \mathrm{~N}-41.5 \mathrm{~N}$ & $14.2 \mathrm{E}-12.5 \mathrm{E}$ & ISIDE-INGV \\
Loma Prieta_Ca, USA & $24 / 09 / 1982-18 / 10 / 1989$ & $38.0 \mathrm{~N}-36.0 \mathrm{~N}$ & $120.0 \mathrm{~W}-123.0 \mathrm{~W}$ & PDE-USGS \\
Turkey & $09 / 04 / 1988-24 / 10 / 2011$ & $44.0 \mathrm{~N}-36.0 \mathrm{~N}$ & $44.0 \mathrm{E}-36.0 \mathrm{E}$ & PDE-USGS \\
Japan & $25 / 09 / 2003-11 / 03 / 2011$ & $42.0 \mathrm{~N}-30.0 \mathrm{~N}$ & $130.0 \mathrm{E}-145.0 \mathrm{E}$ & PDE-USGS \\
Chile & $03 / 09 / 2006-27 / 02 / 2010$ & $30.0 \mathrm{~S}-40.0 \mathrm{~S}$ & $63.0 \mathrm{~W}-80.0 \mathrm{~W}$ & PDE-USGS \\
Samoa & $09 / 01 / 1995-29 / 09 / 2009$ & $12.0 \mathrm{~S}-20.0 \mathrm{~S}$ & $168.0 \mathrm{~W}-176.0 \mathrm{~W}$ & PDE-USGS \\
Sumatra & $01 / 01 / 1981-30 / 04 / 2005$ & $90.0 \mathrm{~S}-130.0 \mathrm{~S}$ & $10.0 \mathrm{E}-15.0 \mathrm{E}$ & PDE-USGS \\
Nepal & $18 / 07 / 2002-12 / 05 / 2015$ & $32.0 \mathrm{~N}-26.0 \mathrm{~N}$ & $88.0 \mathrm{E}-81.0 \mathrm{E}$ & PDE-USGS \\
Tonga & $22 / 03 / 2003-03 / 05 / 2006$ & $16.0 \mathrm{~S}-24.0 \mathrm{~S}$ & $179.0 \mathrm{~W}-178.0 \mathrm{~W}$ & PDE-USGS \\
Russia & $28 / 06 / 1990-05 / 12 / 1997$ & $59.0 \mathrm{~N}-51.0 \mathrm{~N}$ & $170.0 \mathrm{E}-154.0 \mathrm{E}$ & PDE-USGS \\
\hline
\end{tabular}

that have developed before some strong earthquakes happened in the past in various parts of the world.

The branching structures, identified with the long term, seismic sequence hierarchization, show that big earthquakes can trigger additional earthquakes with decreasing magnitude during the energy accumulation stage. After the trigger point formation (yellow triangle), we notice some energy release stages of flash earthquakes (Figures 16 (a)-(e)) or progressive earthquakes type (Figures 16(f)-(I)). Comprehensive retrospective tests show that the proposed methodology is reasonably stable upon changes in the size of the seismic area and therefore it is able to locate all the branching structures that develop during the energy accumulation and release stages.

\section{Conclusions}

This study has the aim to propose a model for the graphical analysis of seismic sequences, starting from the assumption that the sequences do not develop in an unpredictable manner, but follow easily identifiable trends.

As a result, we can say that some developments recur cyclically, making it possible, if identified on time, the formulation of hypotheses on the future development of a seismic sequence.

The proposed model, based on the earthquakes time series' hierarchization, has shown that the seismic sequences develop according to similar developmental stages and that the mainshock magnitude value associated with a seismological branching sequence depends on the first developmental stage (higher order seismic branch).

This approach develops a dramatically innovative earthquake forecasting model starting from the seismicity analysis conducted on an area of different width, which provides a reasonable estimate of the expected magnitude.

\section{References}

[1] Ogata, Y. (1999) Seismicity Analysis through Point-Process Modeling: A Review. Pure and Applied Geophysics, 155, 471-507. http://dx.doi.org/10.1007/s000240050275

[2] Helmstetter, A. and Sornette, D. (2002) Diffusion of Epicenters of Earthquake Aftershocks, Omori’s Law, and Generalized Continuous-Time Random Walk Models. Physical Review E, 66, Article ID: 061104. http://dx.doi.org/10.1103/PhysRevE.66.061104

[3] Gu, C., Schumann, A.Y., Baiesi, M. and Davidsen, J. (2013) Triggering Cascades and Statistical Properties of Aftershocks. Journal of Geophysical Research: Solid Earth, 118, 4278-4295. http://dx.doi.org/10.1002/jgrb.50306

[4] Riga G. and Balocchi, P. (2016) Seismic Sequence Structure and Earthquakes Triggering Patterns. Open Journal of Earthquake Research, 5, No. 1. http://www.scirp.org/Journal/PaperInformation.aspx?PaperID=63538

[5] Parsons, T. and Velasco, A. A. (2009) On Near-Source Earthquake Triggering. Journal of Geophysical Research, 114, Article ID: B10307. http://dx.doi.org/10.1029/2008JB006277

[6] Zhuang, J., Ogata, Y. and Vere-Jones, D. (2002) Stochastic Declustering of Space-Time Earthquake Occurrences. Journal of the American Statistical Association, 97, 369-380. http://dx.doi.org/10.1198/016214502760046925

[7] Zhuang, J., Ogata, Y. and Vere-Jones, D. (2004) Analyzing Earthquake Clustering Features by Using Stochastic Reconstruction. Journal of Geophysical Research, 109, Article ID: B05301. http://dx.doi.org/10.1029/2003JB002879 
[8] Baiesi, M. and Paczuski, M. (2004) Scale-Free Networks of Earthquakes and Aftershocks. Physical Review E, 69, Article ID: 066106. http://dx.doi.org/10.1103/PhysRevE.69.066106

[9] Baiesi, M. and Paczuski, M. (2005) Complex Networks of Earthquakes and Aftershocks. Nonlinear Processes in Geophysics, 12, 1-11. http://dx.doi.org/10.5194/npg-12-1-2005

[10] Zaliapin, I., Gabrielov, A., Keilis-Borok, V. and Wong, H. (2008) Clustering Analysis of Seismicity and Aftershock Identification. Physical Review Letters, 101, Article ID: 018501. http://dx.doi.org/10.1103/PhysRevLett.101.018501

[11] Marsan, D. and Lengliné, O. (2008) Extending Earthquakes’ Reach through Cascading. Science, 319, 1076-1079. http://dx.doi.org/10.1126/science.1148783

[12] Mogi, K. (1963) Some Discussions on Aftershocks, Foreshocks and Earthquake Swarms-The Fracture of a SemiInfinite Body Caused by an Inner Stress Origin and Its Relation to the Earthquake Phenomena (Third Paper). Bulletin of the Earthquake Research Institute, 41, 615-658.

[13] Van Stiphout, T., Wiemer, S. and Marzocchi, W. (2010) Are Short-Term Evacuations Warranted? Case of the 2009 L’Aquila Earthquake. Geophysical Research Letters, 37, L06306. http://dx.doi.org/10.1029/2009gl042352

[14] Jones, L.M. and Molnar, P. (1976) Frequency of Foreshocks. Nature, 262, 677-679. http://dx.doi.org/10.1038/262677a0

[15] Abercrombie, R.E. and Mori, J. (1996) Occurrence Patterns of Foreshocks to Large Earthquakes in the Western United States. Nature, 381, 303-307. http://dx.doi.org/10.1038/381303a0

[16] Keilis-Borok, V.I. and Malinovskaya, L.N. (1964) One Regularity in the Occurrence of Strong Earthquakes. Journal of Geophysical Research, 69, 3019-3024.

[17] Jaumé, S.C. and Sykes, L.R. (1999) Evolving Towards a Critical Point: A Review of Accelerating Seismic Moment/ Energy Release Prior to Large and Great Earthquakes. In: Wyss, M., Shimazaki, K. and Ito, A., Eds., Seismicity Patterns, Their Statistical Significance and Physical Meaning, Birkhäuser, Basel, 279-305.

http://link.springer.com/chapter/10.1007\%2F978-3-0348-8677-2 5 http://dx.doi.org/10.1007/978-3-0348-8677-2__5

[18] Sammis, S.G. and Sornette, D. (2002) Positive Feedback, Memory and the Predictability of Earthquakes. Proceedings of the National Academy of Sciences of the United States of America, 99, 2501-2508. http://arxiv.org/pdf/cond-mat/0107143v1.pdf http://dx.doi.org/10.1073/pnas.012580999

[19] Ishibashi, K. (1988) Two Categories of Earthquake Precursors, Physical and Tectonic, and Their Roles in IntermediateTerm Earthquake Prediction. Pure and Applied Geophysics, 126, 687-700. http://dx.doi.org/10.1007/bf00879015

[20] Shebalin, P. (2006) Increased Correlation Range of Seismicity before Large Events Manifested by Earthquake Chains. Tectonophysics, 424, 335-349. http://dx.doi.org/10.1016/j.tecto.2006.03.040

[21] Mantovani, E., Babbucci, D., Tamburelli, C. and Viti, M. (2009) A Review on the Driving Mechanism of the Tyrrhenian-Apennines System: Implications for the Present Seismotectonic Setting in the Central-Northern Apennines. Tectonophysics, 476, 22-40. http://dx.doi.org/10.1016/j.tecto.2008.10.032

[22] Ziv, A. and Schmittbuhl, J. (2003) The Seismic Cycle and the Difference between Foreshocks and Aftershocks in a Mechanical Fault Model. Geophysical Research Letters, 30, 2237. https://eost.unistra.fr/fileadmin/upload/EOST/Jean_Schmittbuhl/Publications/Articles/Zi-Sc03.pdf

[23] Helmstetter, A. and Sornette, D. (2003) Foreshocks Explained by Cascades of Triggered Seismicity. Journal of Geobphysical Research: Solid Earth, 108, 2457.

[24] Helmstetter, A., Kagan, Y.Y. and Jackson, D.D. (2005) Importance of Small Earthquakes for Stress Transfers and Earthquake Triggering. Journal of Geophysical Research: Solid Earth, 110, Article ID: B05S08. http://onlinelibrary.wiley.com/doi/10.1029/2004JB003286/full

[25] Shearer, P.M. (2012) Space-Time Clustering of Seismicity in California and the Distance Dependence of Earthquake Triggering. Journal of Geophysical Research: Solid Earth, 117, B10306. http://dx.doi.org/10.1029/2012jb009471

[26] Helmstetter, A. (2003) Is Earthquake Triggering Driven by Small Earthquakes? Physical Review Letters, 91, Article ID: 058501. http://dx.doi.org/10.1103/PhysRevLett.91.058501

[27] Felzer, K.R. and Brodsky, E.E. (2006) Decay of Aftershock Density with Distance Indicates Triggering by Dynamic Stress. Nature, 441, 735-738. http://dx.doi.org/10.1038/nature04799

[28] Marzocchi, W. and Lombardi, A.M. (2008) A Double Branching Model for Earthquake Occurrence. Journal of Geophysical Research: Solid Earth, 113, Article ID: B08317. http://dx.doi.org/10.1029/2007JB005472 http://onlinelibrary.wiley.com/doi/10.1029/2007JB005472/pdf

[29] Werner, M.J. (2008) On the Fluctuations of Seismicity and Uncertainties in Earthquake Catalogs: Implications and 
Methods for Hypothesis Testing. University of California, Los Angeles.

https:/www.ethz.ch/content/dam/ethz/special-interest/mtec/chair-of-entrepreneurial-risks-dam/documents/dissertation/ Max Werner thesis final Dec07.pdf

[30] Chande, T. (1995) A Time Price Oscillator. Technical Analysis of Stocks \& Commodities, 13, 369-374. ftp://80.240.216.180/Transmission/\%D0\%A4\%D0\%B0\%D0\%B9\%D0\%BB\%D1\%8B/S\&C\%20on\%20DVD\%2011.26 /VOLUMES/V13/C09/THETIME.pdf 